\title{
Dietary fatty acid profiles and percentage fat-free mass: Cross-sectional associations in the EPIC-Norfolk cohort
}

\author{
R.P.G. Hayhoe ${ }^{1}$, M.A.H. Lentjes ${ }^{2}$, A.A. Mulligan ${ }^{2}$, R.N. Luben ${ }^{2}$, K-T. Khaw ${ }^{2}$ and A.A. Welch ${ }^{1}$ \\ ${ }^{1}$ Population Health and Primary Care, Norwich Medical School, University of East Anglia, Norwich, Norfolk NR4 \\ $7 T J$ and \\ ${ }^{2}$ Public Health and Primary Care, University of Cambridge, Cambridgeshire CB1 8RN
}

Loss of muscle mass and function progresses with age over 30 years, but accelerates in later life and may result in sarcopenia. This condition has been associated with debilitating consequences including physical disability, risk of frailty and falls, and thus a greater requirement for health and social care ${ }^{(1)}$. A number of modifiable lifestyle behaviours, including nutrition and diet, may affect muscle mass and function in older individuals ${ }^{(2)}$. Dietary fat provides a significant source of energy for muscle and the balance of different fatty acids may have effects on inflammatory signalling and insulin resistance potentially involved in sarcopenia progression. Indeed, there is already some evidence of associations between fatty acid intakes and muscle measures in women ${ }^{(3)}$. The aim of this study was to investigate the associations of dietary fatty acid intake and bioelectrical impedance estimated fat-free mass (as a proxy for skeletal muscle mass) in a UK population cohort of older men and women.

Analyses were conducted using data from the EPIC-Norfolk cohort of 25,639 individuals ${ }^{(4)}$. Dietary nutrient intakes were estimated from 7-day food diaries and fat-free mass by bioelectrical impedance analysis (BIA) using a Tanita TBF531 scale. Multivariable regression (using STATA v13; Stata Corp., USA) was used to investigate percentage fat-free mass (FFM $\%$; fat free mass $\div$ total mass $\times 100)$ across sex-specific dietary intake quintiles $(\mathrm{Q})$ of total fat, mono-unsaturated fatty acids (MUFA), poly-unsaturated fatty acids (PUFA), saturated fatty acids (SFA), and the ratio of PUFA to SFA; all as percentages of total energy intake (en\%).

In this population of older individuals (mean (SD) age 62.9 (9.0) years for men $(n=6350)$ and $61.5(9.0)$ for women $(n=7990)$ ), the mean (SD) FFM\% was $76.7(5.8) \%$ for men and $60.9(8.3) \%$ for women. Significantly lower adjusted FFM\% was seen in Q5 vs. Q1 for total fat and MUFA dietary intake, for both men and women, and SFA intake for men alone (see table). Although no associations of adjusted FFM\% with dietary PUFA intake were evident, a positive association was seen with dietary PUFA:SFA intake for both sexes (see table).

\begin{tabular}{|c|c|c|c|c|c|c|c|c|c|c|}
\hline \multirow{2}{*}{\multicolumn{2}{|c|}{ Adjusted $^{\mathrm{a}}$ FFM\% $\%$}} & \multicolumn{2}{|c|}{ Q1 } & \multicolumn{2}{|c|}{ Q3 } & \multicolumn{2}{|c|}{ Q5 } & \multirow{2}{*}{$\begin{array}{l}\text { Q5-1 } \\
\% \text { diff }\end{array}$} & \multirow{2}{*}{$\begin{array}{l}\mathrm{P} \\
\operatorname{diff}^{\mathrm{b}}\end{array}$} & \multirow[b]{2}{*}{$\mathrm{P}$ trend } \\
\hline & & Mean & $\mathrm{SE}$ & Mean & SE & Mean & SE & & & \\
\hline \multirow[t]{5}{*}{ Men } & Total Fat $(\mathrm{en} \%)$ & 76.96 & $0 \cdot 16$ & $76 \cdot 92$ & $0 \cdot 16$ & $76 \cdot 34$ & $0 \cdot 16$ & $-0 \cdot 81$ & 0.007 & 0.004 \\
\hline & MUFA (en $\%)$ & $76 \cdot 95$ & $0 \cdot 16$ & $76 \cdot 93$ & $0 \cdot 16$ & $76 \cdot 15$ & $0 \cdot 16$ & -1.04 & $<0.001$ & $<0.001$ \\
\hline & PUFA (en $\%)$ & $76 \cdot 54$ & $0 \cdot 16$ & $76 \cdot 66$ & $0 \cdot 16$ & $76 \cdot 93$ & $0 \cdot 16$ & $0 \cdot 51$ & 0.082 & $0 \cdot 195$ \\
\hline & SFA $(\mathrm{en} \%)$ & $77 \cdot 12$ & $0 \cdot 16$ & $76 \cdot 60$ & $0 \cdot 16$ & $76 \cdot 49$ & $0 \cdot 16$ & $-0 \cdot 82$ & 0.007 & 0.023 \\
\hline & PUFA:SFA & $76 \cdot 55$ & $0 \cdot 16$ & $76 \cdot 71$ & $0 \cdot 16$ & $77 \cdot 20$ & $0 \cdot 16$ & $0 \cdot 86$ & 0.004 & 0.004 \\
\hline \multirow[t]{5}{*}{ Women } & Total Fat $(\mathrm{en} \%)$ & 61.45 & $0 \cdot 21$ & 61.09 & $0 \cdot 20$ & $60 \cdot 60$ & $0 \cdot 21$ & -1.40 & $0 \cdot 005$ & 0.008 \\
\hline & MUFA (en $\%)$ & $61 \cdot 40$ & $0 \cdot 21$ & $60 \cdot 87$ & $0 \cdot 20$ & $60 \cdot 48$ & $0 \cdot 20$ & $-1 \cdot 50$ & $0 \cdot 002$ & $<0.001$ \\
\hline & PUFA (en $\%)$ & $61 \cdot 12$ & $0 \cdot 20$ & $60 \cdot 72$ & $0 \cdot 20$ & $61 \cdot 11$ & $0 \cdot 20$ & $0 \cdot 00$ & 0.992 & $0 \cdot 307$ \\
\hline & SFA $(e n \%)$ & $61 \cdot 31$ & $0 \cdot 21$ & $60 \cdot 65$ & $0 \cdot 20$ & $60 \cdot 73$ & $0 \cdot 21$ & -0.95 & 0.055 & 0.083 \\
\hline & PUFA:SFA & $60 \cdot 84$ & $0 \cdot 20$ & $60 \cdot 77$ & $0 \cdot 20$ & 61.47 & $0 \cdot 20$ & 1.04 & 0.029 & 0.035 \\
\hline
\end{tabular}

a Adjusted for: age, smoking status, physical activity, social class, menopausal and HRT status in women, statin use, corticosteroid use, days of food diary completed, total energy intake, and protein energy intake as a percentage of total. ${ }^{\mathrm{b}} \mathrm{P}$ value for absolute difference between Q5 vs. Q1, according to ANCOVA.

This study has shown novel evidence that dietary fatty acid intakes are significantly associated with a measure of skeletal muscle mass in both men and women. In particular the balance between PUFA and SFA dietary intakes appears to be important. Higher PUFA at equal quantities of SFA was associated with higher FFM $\%$ in this cohort. Further mechanistic analyses and controlled trials are required, but these data nevertheless suggest that optimising fatty acid dietary profiles may have utility as part of a sarcopenia prevention strategy.

1. Welch A (2014) Proc Nut Soc 73, 16-33.

2. Denison H, Cooper C, Sayer A, Robinson S (2015) Clin Interv Aging 10, 859-69.

3. Welch A, MacGregor A, Minihane A et al. (2014) J Nutr 144(3): 327-34.

4. Day N, Oakes S, Luben R et al. (1999) Br J Cancer 80, 95-103. 